\title{
On the dependence of crack surface morphology and energy dissipation on microstructure in ductile plate tearing
}

Çelik; Andersen, R. G.; Tekolu, C.; Nielsen, K. L.

Published in:

International Journal of Fracture

Link to article, DOI:

$10.1007 / \mathrm{s} 10704-020-00513-8$

Publication date:

2021

Document Version

Peer reviewed version

Link back to DTU Orbit

Citation (APA):

Celik, Andersen, R. G., Tekolu, C., \& Nielsen, K. L. (2021). On the dependence of crack surface morphology and energy dissipation on microstructure in ductile plate tearing. International Journal of Fracture, 230, 115-132 . https://doi.org/10.1007/s10704-020-00513-8

\section{General rights}

Copyright and moral rights for the publications made accessible in the public portal are retained by the authors and/or other copyright owners and it is a condition of accessing publications that users recognise and abide by the legal requirements associated with these rights.

- Users may download and print one copy of any publication from the public portal for the purpose of private study or research.

- You may not further distribute the material or use it for any profit-making activity or commercial gain

- You may freely distribute the URL identifying the publication in the public portal 


\title{
On the Dependence of Crack Surface Morphology and Energy Dissipation on Microstructure in Ductile Plate Tearing
}

\author{
Ş. Çelik ${ }^{\mathrm{a}}$, R. G. Andersen ${ }^{\mathrm{b}}$, C. Tekoğlu ${ }^{\mathrm{a}, *}$, K. L. Nielsen ${ }^{\mathrm{b}}$ \\ ${ }^{a}$ Department of Mechanical Engineering, TOBB University of Economics and Technology, Sög̃ütözü, \\ Ankara, 06560, Turkey \\ ${ }^{b}$ Department of Mechanical Engineering, Solid Mechanics, Technical University of Denmark, \\ DK-2800 Kgs. Lyngby, Denmark
}

\begin{abstract}
The two distinct tearing mechanisms observed in ductile metal plates are the void-byvoid advance of the crack tip, and the simultaneous interaction of multiple voids on the plane ahead of the crack tip. Void-by-void crack advance, which leads to a cupcup crack surface morphology, is the dominant mechanism if the plate contains a low number of small void nucleation sites (i.e., second phase particles). Conversely, a large number and/or size of nucleation sites trigger the simultaneous interaction of multiple voids resulting in a slanted crack. The present work aims to provide further insight into the parameters controlling the mechanisms and energy dissipation of plate tearing by focusing on the shape and, thereby, the orientation of the nucleation sites. The study uses a two-dimensional plane strain finite element domain to model the cross section of a plate, subject to mode I tearing, with discretely modeled, randomly distributed, finite-sized elliptic void nucleation sites. The developed finite element setup can capture the dependence of the crack surface morphology on the microstructure of the plate. The simulation results confirm that cup-cup crack propagation develops by intense plastic straining throughout the thinning region of the plate. Conversely, slanted and cup-cone cracks propagate in thin localized shear deformation bands. The energy dissipation is, therefore, greater for cup-cup cracks. The study demonstrates that the damage-related microstructure has a significant role in determining the overall hardening capacity of a plate, which in turn dictates the tearing mode and energy.
\end{abstract}

Keywords: Ductile failure, Gurson model, Void interaction, Size effect, Shape effect

*Corresponding author. Tel.:+90 3122924229 ; fax:+90 3122924091 .

URL: cihantekoglu@etu.edu.tr; c.tekoglu@gmail.com (C. Tekoğlu) 


\section{Introduction}

The fracture toughness and crack resistance (or energy dissipation) related to largescale plate tearing are crucial to the integrity of a structure and therefore, of significant industrial interest. Centuries of material research have successfully improved the performance of metal plates by exploiting alloying elements and phase transformations to block, partially or entirely, the dislocation movement required to inflict permanent (plastic) deformation. Srivastava et al. (2017) recently applied a new approach to enhance the fracture toughness and numerically engineered the crack path by exploiting the ductile failure sequence of void nucleation and growth to coalescence (also building on the work in Srivastava et al., 2014). By tailoring the location of the void nucleation sites in the material, Srivastava and co-workers managed a fracture toughness two to three times greater than that of a plate material with randomly distributed nucleation sites of identical mean spacing. The increase in toughness is impressive and links to the extent of the fracture process zone and widespread plastic dissipation (the energy involved is orders of magnitude greater than that of atomic separation). The high toughness values were achieved by arranging void nucleation sites on a sinusoidal path in front of a pre-notch, and tuning the amplitude to wavelength ratio to favor the multiple void interaction mechanisms demonstrated by Tvergaard and Hutchinson (2002). At a critical ratio, the tearing crack advances along the prescribed sinusoidal path by the interaction of the embedded nucleation sites. Conversely, the crack jumps from one crest (or trough) to another in a void-by-void-like mechanism for ratios deviating substantially from the critical ratio. Despite its fundamental importance, it is difficult to apply the approach by Srivastava et al. (2017) to large-scale engineering structures.

The present work considers a material with randomly distributed nucleation sites and focuses on the microstructure configuration to investigate crack growth resistance under steady-state tearing. The hypothesis is that the tearing mode and associated energy dissipation, under mode I loading conditions, can be controlled by the volume fraction, size, shape, and orientation of the void nucleation sites. This study, thereby, follows the idea of Srivastava and co-workers, and exploits the interaction mechanisms 
to enhance the material performance. However, here, this is on a length scale of (at least) one order of magnitude greater than the mean void spacing. In the terminology of Pardoen et al. (2004), the present study shifts the focus to the extrinsic energy dissipation related to plate thinning (or necking) in the fracture process zone, which scales with the plate thickness (rather than the intrinsic energy, scaling with the void spacing). Recently, Andersen et al. (2020) demonstrated, through numerical simulations, a tight connection between the damage-related microstructure and extrinsic energy dissipation during extensive crack growth. An increase in either the number or size of the discretely modeled, randomly distributed, circular void nucleation sites decreases the mean energy dissipation without a noticeable effect on the peak strength. The main reason for the decrease in the energy is a shift from a cup-cup-like tearing mode, relying on severe plate thinning, to a slant failure that utilizes macroscopic shear localization and limited plate thinning. Investigations indicate that, when randomly distributed in a plate, a small number of nucleation sites promote cup-cup (also referred to as flat) failure, whereas a large number of nucleation sites, or even, a small number of large sites, yield a slant failure (see Tekoğlu and Nielsen, 2019). The shift ties to the overall hardening capacity of the plate material; Pardoen et al. (2004) discuss this in detail in relation to the cup-cup tearing mode for a broad palette of materials with moderate to high overall hardening capacity (see also Pineau et al., 2016). Similarly, materials with low overall hardening capacity typically display a flat-to-slant transition after the crack has propagated on the order of one plate thickness, and subsequently propagate in a slant configuration with limited plate thinning (see Hickey and Ravi-Chandar, 2015).

Heat treatments and/or deformation processes, in combination with optimized alloying components, offer a wide control over the structure of the second phase particles acting as void nucleation sites. These numerical findings should serve as a guideline for material scientists. The intent of the present work is to identify the configurations of the material microstructures to target in the manufacturing process to achieve specific tearing characteristics. Moreover, the work clarifies why certain plate tearing experiments display cup-cup (or bath-tub) crack propagation, whereas others yield tearing by slanting cracks during mode I loading. The model setup predicts the two fracture morphologies, consistently, for extreme cases of the damage-related microstructure; 
however, the transition between the morphologies is gradual owing to the randomness invoked by the distribution of the nucleation sites. A two-dimensional (2D) plane strain model setup approximates the ductile tearing process such that only a cross section of the plate material in front of the propagating crack is considered (see also Nielsen and Hutchinson, 2012; Andersen et al., 2019).

The paper has the following structure. Section 2 presents the problem formulation, modeling assumptions, and numerical methods. The results are presented in Section 3, with the related discussion provided in Section 4. Section 5 presents the conclusions.

\section{Problem formulation and finite element model}

The authors of the present paper have recently investigated how the number and relative size of randomly distributed circular second phase particles (i.e., void nucleation sites) influence the crack surface morphology (see Tekoğlu and Nielsen, 2019) and energy dissipation (see Andersen et al., 2020) in ductile tearing of thin metal plates. The present study extends the previous works by allowing the void nucleation sites to assume elliptical shapes, whereby their orientation must also be considered. The formulation of ductile plate tearing and the finite element (FE) model developed to address this problem are briefly explained below. For further details, the reader is referred to Tekoğlu and Nielsen (2019); Andersen et al. (2020).

\subsection{Problem formulation}

The schematics in Figs. 1(a) and (b) illustrate different stages of crack propagation in a ductile metal plate that tears under mode I loading ${ }^{1}$. In the undeformed (initial) configuration, the plate is assumed to have in-plane dimensions considerably larger than the plate thickness, and the crack to have settled into steady-state tearing. At stage (1) in the tearing process, depicted in Figs. 1(a) and (b), diffuse thinning of the plate section starts significantly ahead of the crack tip and sets the extension of the fracture process zone. From this point onward, the interaction and evolution of the different stages leading to crack advance depend on the overall hardening capacity for

\footnotetext{
${ }^{1}$ Figure 1(a) illustrates the crack growth by the void-by-void mechanism and Fig. 1(b) by the simultaneous interaction of multiple voids. A discussion follows in Section 4.
} 
the plate section set by the material configuration, which also determines the crack surface morphology (see Tekoğlu and Nielsen, 2019). Imagine following a cross section of the plate from initiation of the plate thinning, i.e., when it enters the fracture process zone, until final separation. In this sequence of events, all cross sections experience the same loading history at steady-state. Moreover, the cross sections of the fracture process zone are well approximated in the 2D plane strain (neglecting gradients in the crack growth direction) as the material above and below the thinning region elastically unloads and imposes a constraint on the deformation in the crack growth direction (see Andersen et al., 2019). A 2D modeling approach to large-scale plate tearing was used in Nielsen and Hutchinson (2012) to study isotropic metals, and later extended to heterogeneous materials in Tekoğlu and Nielsen (2019). The present work further extends the investigation of large-scale plate tearing by adopting the $2 \mathrm{D}$ plane strain setup; it considers the shape and orientation of the void nucleation sites in addition to their number and size (see Fig. (c)). The nucleation sites are randomly distributed within the fracture process zone (see also Tekoğlu and Nielsen, 2019).

A cross section with the height of the plate being four times the initial plate thickness (i.e., $H_{0}=4 W_{0}$ ) is considered with a fracture process zone of initial height $h_{0}=2 W_{0}$, see Fig. 1(c). The fracture process zone consists of a strain hardening matrix material that contains a small background porosity (governed by strain-controlled nucleation) to allow cracks to propagate. Embedded in the fracture process zone are a number, $N_{\mathrm{p}}$, of void nucleation sites, discretized by several finite elements and governed by strain-controlled nucleation to mimic the nucleation of voids within the void nucleation sites (see the material properties in Table 1). Figure 1(c) illustrates the shape of the nucleation sites and their orientation angle, $\psi$, relative to the main loading direction. Throughout, all sites are assumed to be elliptical with identical aspect ratios, $R$ (being the ratio of the major axis to the minor axis) and orientations, $\psi$. The size of the void nucleation sites is determined by the ratio $R_{\mathrm{p}} / L_{\mathrm{e}}$, where $L_{\mathrm{e}}$ is the edge length of the initially square finite elements (of size $L_{\mathrm{e}} \times L_{\mathrm{e}}$ ) discretizing the fracture process zone, and $R_{\mathrm{p}}$ is the radius of a corresponding circular nucleation site with the same area. In order to generate a void nucleation site, the center of a randomly selected element in the fracture process zone is taken to be the center of the (hypothetical) 
ellipse that encloses the nucleation site (see the ellipse with a dashed line type in Fig. 1(c)). The randomly selected central element and all its closest neighboring elements, whose centers are in the ellipse, form the nucleation site. The total number of elements is 29 for a nucleation site with a size of $R_{\mathrm{p}} / L_{\mathrm{e}}=3,113$ for $R_{\mathrm{p}} / L_{\mathrm{e}}=6$, and 253 for $R_{\mathrm{p}} / L_{\mathrm{e}}=9$. Note that for $R_{\mathrm{p}} / L_{\mathrm{e}}=6$ (respectively, $R_{\mathrm{p}} / L_{\mathrm{e}}=9$ ), the area of a nucleation site should in fact be 4 (respectively, 9) times larger than that for $R_{\mathrm{p}} / L_{\mathrm{e}}=3$, i.e. the nucleation site should contain 116 (respectively, 261) elements. It is, however, impossible to fit the exact numbers of square elements in elliptical nucleation sites. For the very same reason, the nucleation sites with $R_{\mathrm{p}} / L_{\mathrm{e}}=6, A=5$, and $\psi=\left\{0^{\circ}, 90^{\circ}\right\}$, (respectively, $R_{\mathrm{p}} / L_{\mathrm{e}}=9, R=5$, and $\left.\psi=\left\{0^{\circ}, 90^{\circ}\right\}\right)$ the total number of elements is 115 (respectively, 255). It was assumed that the individual void nucleation sites are nonoverlapping and do not intersect the cross-section boundaries. The minimum allowable distance between the boundaries of two neighboring void nucleation sites, and between the boundary of a void nucleation site and the cross-section boundaries was assigned to be one element. To ensure a (more or less) elliptical shape, the largest aspect ratio that can be assigned to a nucleation site of size $R_{\mathrm{p}} / L_{\mathrm{e}}=3$ is $R=3$. Therefore, $R$ is considered to be equal to 1 or 3 for $R_{\mathrm{p}} / L_{\mathrm{e}}=3$, whereas $R=\{1,5,10\}$ for $R_{\mathrm{p}} / L_{\mathrm{e}}>3$. All possible $\left(N_{\mathrm{p}}, R_{\mathrm{p}} / L_{\mathrm{e}}, R, \psi\right)$ combinations are analyzed, and three realizations with different spatial distributions of nucleation sites are considered for each combination (in total $162 \mathrm{FE}$ simulations are made).

The 2D plane strain cross section is loaded in tension by prescribing the displacement along the $x_{2}$-axis, whereas the reaction force is extracted along the top of the domain (at $x_{2}=H_{0}$ ). The overall engineering stress on the plate cross section is then calculated as the sum of forces in the main loading direction divided by the initial surface area of the plate: $\Sigma_{22}=\sum_{i=1}^{n} F_{2}^{i} / A_{0}$. The relative change of the height of the plate provides the work conjugate engineering strain: $E_{22}=\Delta H / H_{0}$. The energy dissipation is of primary interest and is calculated as the area under the load-deflection curve from where the $\Delta H / H_{0}=N$ (which approximates the attainment of the peak force, $F_{\text {peak }}$ ), until final separation (see Fig. 1(d)). The dissipated energy is denoted by $\Gamma_{0}$. 


\subsection{Constitutive model: Gurson material}

The present work adopts the Gurson-Tvergaard-Needleman (GTN) model (Gurson, 1977; Tvergaard, 1981; Tvergaard and Needleman, 1984) to enable void nucleation and growth to coalescence within the fracture process zone. The yield surface for this classical model has the following form

$$
\Phi=\left(\frac{\sigma_{\mathrm{e}}}{\sigma_{\mathrm{M}}}\right)^{2}+2 q_{1} f^{*} \cosh \left(\frac{3 q_{2}}{2} \frac{\sigma_{\mathrm{m}}}{\sigma_{\mathrm{M}}}\right)-\left(1+\left(q_{1} f^{*}\right)^{2}\right),
$$

and is based on the effective von Mises stress given as $\sigma_{\mathrm{e}}=\sqrt{3 s_{i j} s_{i j} / 2}$, where $s_{i j}$ are the deviatoric components of the Cauchy stress tensor. The instantaneous yield stress of the fully dense material is denoted as $\sigma_{\mathrm{M}}, \sigma_{\mathrm{m}}=\sigma_{k k} / 3$ is the mean hydrostatic stress, and $q_{1}$ and $q_{2}$ are the Tvergaard constants (see Tvergaard, 1981). Void coalescence is incorporated through the effective void volume fraction, $f^{*}$, which accelerates damage evolution when the void volume fraction achieves a critical value, $f_{\mathrm{C}}$. The coalescence model thereby has the form

$$
f^{*}= \begin{cases}f & \text { for } f \leq f_{\mathrm{C}}, \\ f_{\mathrm{C}}+\frac{\bar{f}_{\mathrm{F}}-f_{\mathrm{C}}}{f_{\mathrm{F}}-f_{\mathrm{C}}}\left(f-f_{\mathrm{C}}\right) & \text { for } f>f_{\mathrm{C}}\end{cases}
$$

with $\bar{f}_{\mathrm{F}}=1 / q_{1} ; f_{\mathrm{F}}$ and $f_{\mathrm{C}}$ are the critical and final void volume fraction, respectively. The void volume fraction of the material, $f$, is assumed to develop partly owing to the growth of the existing voids, $\dot{f}_{\text {growth }}$, and partly because of the nucleation of the damage, $\dot{f}_{\text {nucl }}$, such that $\dot{f}=\dot{f}_{\text {growth }}+\dot{f}_{\text {nucl }}$. The contribution from the void growth follows directly from the plastic incompressibility, $\dot{f}_{\text {growth }}=(1-f) \dot{\varepsilon}_{k k}^{\mathrm{pl}}$. The two distinct void nucleation mechanisms typically occurring in metals (and metal alloys) are the fracture of second phase particles and decohesion of the interface between the particle and the surrounding matrix material (e.g., Gurland, 1972; Cox and Low, 1974; Marino et al., 1985; Lewandowski et al., 1989; Dighe et al., 2002; Bron et al., 2004; AsserinLebert et al., 2005; Tekoğlu and Pardoen, 2010). The nucleation of voids is assumed to evolve with the microscopic equivalent plastic strain, $\varepsilon_{M}^{p}$, following the Gaussian-bell nucleation law by Chu and Needleman (1980), such that

$$
\dot{f}_{\text {nucl }}=\frac{f_{\mathrm{N}}}{s_{\mathrm{N}} \sqrt{2 \pi}} \exp \left[-\frac{1}{2}\left(\frac{\varepsilon_{\mathrm{M}}^{\mathrm{pl}}-\varepsilon_{\mathrm{N}}}{s_{\mathrm{N}}}\right)^{2}\right] \dot{\varepsilon}_{\mathrm{M}}^{\mathrm{pl}},
$$


where the Gaussian bell curve resembles a statistical variation in the void nucleation process of a total potential void volume fraction, $f_{\mathrm{N}}$. The mean nucleation strain is denoted by $\varepsilon_{\mathrm{N}}$, and $s_{\mathrm{N}}$ is the standard deviation.

For the clarity of the results, the matrix material is assumed to follow the power-law stress-strain relation in uniaxial tension,

$$
\sigma= \begin{cases}E \varepsilon & \text { for } \varepsilon<\varepsilon_{0}, \\ \sigma_{0}\left(\frac{\varepsilon}{\varepsilon_{0}}\right)^{N} & \text { for } \varepsilon \geq \varepsilon_{0} .\end{cases}
$$

Here, $E$ is the Young's modulus, $\varepsilon_{0}$ denotes the yield strain, and $N$ is the strain hardening exponent. All material parameter values are listed in Table 1.

\subsection{Finite element (FE) model}

Different layouts of the damage-related microstructure embedded within the plate cross section outlined in Fig. 1(c) are analyzed numerically using the commercial FE software package Abaqus/Explicit (Dassault Systèmes Simulia Corp., 2016) in a finite strain setting. The spatial discretization in the fracture process zone consists of four-node bilinear plane strain elements (CPE4R) with a square shape in the undeformed configuration. The initial edge length of the square-shaped elements is $L_{e} / W_{0}=1 / 800$. Outside the fracture process zone, three-node linear elements are allowed (CPE3 elements according to Dassault Systèmes Simulia Corp., 2016), and the mesh is made coarser towards the top and bottom boundaries of the cross section to limit the calculation time spent on each simulation. The material outside the fracture process zone is governed by the $J_{2}$ plasticity. All simulations are conducted within a dynamic framework, yet with the load applied sufficiently slowly to assume quasi-static conditions. Care has been taken that the kinetic energy is considerably less than $10 \%$ of the total energy in the system.

\section{Results}

An extensive parametric study was performed to investigate the effects of microstructural heterogeneity (i.e., the size, shape, orientation, and spatial distribution of the void nucleation sites) on the crack surface morphology and energy dissipation in ductile mode I plate tearing. The sections below present representative results that identify 
Table 1: Material Properties

\begin{tabular}{lcc}
\hline Parameters & Notation & Value \\
\hline Density & $\rho$ & $2700 \mathrm{~kg} / \mathrm{m}^{3}$ \\
Young's modulus & $E$ & $70 \mathrm{GPa}$ \\
Poisson's ratio & $v$ & 0.3 \\
Yield stress & $\sigma_{0}$ & $300 \mathrm{MPa}$ \\
Strain hardening exponent & $N$ & 0.1 \\
Gurson fitting parameters & $q_{1}, q_{2}$ & $1.5,1.0$ \\
Void volume fraction that can potentially nucleate & $f_{\mathrm{N}}^{\mathrm{ns}}, f_{\mathrm{N}}^{\mathrm{m}}$ & $8 \times 10^{-3}, 1 \times 10^{-5}$ \\
Mean strain for nucleation & $\varepsilon_{\mathrm{N}}^{\mathrm{ns}}, \varepsilon_{\mathrm{N}}^{\mathrm{m}}$ & $1 \times 10^{-2}$ \\
Standard deviation for mean strain & $s_{\mathrm{N}}^{\mathrm{ns}}, s_{\mathrm{N}}^{\mathrm{m}}$ & $(1 / 3) \times 10^{-2}$ \\
Critical void volume fraction & $f_{\mathrm{C}}^{\mathrm{ns}}, f_{\mathrm{C}}^{\mathrm{m}}$ & $1 \times 10^{-2}$ \\
Void volume fraction at failure & $f_{\mathrm{F}}^{\mathrm{ns}}, f_{\mathrm{F}}^{\mathrm{m}}$ & $5 \times 10^{-2}$ \\
\hline
\end{tabular}

The superscript "ns" refers to the "void nucleation sites" and "m" refers to the "matrix" material surrounding the nucleation sites.

Table 2: Volume (area) fraction of void nucleation sites in undeformed (initial) configuration of plate.

\begin{tabular}{lccc}
\hline & \multicolumn{3}{c}{$N_{\mathrm{p}}$} \\
\cline { 2 - 4 }$R_{\mathrm{p}} / L_{\mathrm{e}}$ & 10 & 25 & 100 \\
\hline 3 & $2.90 \times 10^{-4}$ & $7.25 \times 10^{-4}$ & $2.90 \times 10^{-3}$ \\
6 & $1.13 \times 10^{-3}$ & $2.83 \times 10^{-3}$ & $1.13 \times 10^{-2}$ \\
9 & $2.53 \times 10^{-3}$ & $6.33 \times 10^{-3}$ & $2.53 \times 10^{-2}$
\end{tabular}

the transition between different crack surface morphologies and the associated energy required to advance the crack. The results for circular nucleation sites are included here for comparison purposes; the reader is referred to Tekoğlu and Nielsen (2019) for a complete investigation.

\subsection{Crack surface morphology of plates with heterogeneous microstructures}

In this section, the following values were considered for the key parameters in the FE calculations: $N_{\mathrm{p}}=\{10,25,100\}, R_{\mathrm{p}} / L_{\mathrm{e}}=\{3,6,9\}, R=\{1,3,5,10\}$, and $\psi=$ $\left\{0^{\circ}, 45^{\circ}, 90^{\circ}\right\}$; all other material and geometrical parameters were fixed. The strain 
hardening exponent of the matrix material was $N=0.10$. As outlined in Section 2, while varying $R$ and $\psi$, for a given combination of $N_{\mathrm{p}}, R_{\mathrm{p}} / L_{\mathrm{e}}$, the size (area) of the void nucleation sites was maintained constant such that the initial volume (area) fraction of the nucleation sites remained unchanged and independent of $R$ and $\psi$, see Table 2 .

Figures 2(a)-(d) display the distribution of the void volume fraction in four different plates loaded until final failure, and Fig. 2(e) indicates the corresponding engineering stress-strain curves (defined in Section 2). For all four plates, $N_{\mathrm{p}}=10$ and $R_{\mathrm{p}} / L_{\mathrm{e}}=3$; the void nucleation sites were circular in (a), had an aspect ratio-orientation angle combination of $R=3-\psi=0^{\circ}$ in (b), $R=3-\psi=45^{\circ}$ in (c), and $R=3-\psi=90^{\circ}$ in (d). The crack surface morphologies in Figs. 2(a), (b) and (c) are all in a cup-cup form, where the top and bottom sides of the crack surfaces are virtually mirror images of each other. The main loading direction was perpendicular to the plane of separation for a cup-cup crack. The plate displayed in Fig. 2(d), conversely, yielded crack propagation with a slanted morphology, where separation has occurred in an approximately $45^{\circ}$ band (ideally posing $180^{\circ}$ rotational symmetry) with respect to the main loading direction. The stress-strain curves for all four plates essentially coincide until the onset of the macroscopic localization of the plastic flow (indicated by an asterisk (*) in Fig. $2(\mathrm{e}))^{2}$. However, the orientation of the void nucleation sites strongly influences the subsequent localization and fracture. When arranged in ascending order according to their overall strain at the onset of the macroscopic localization, the order of the four plates is (d) $90^{\circ}$, (c) $45^{\circ}$, (a) circular, and (b) $0^{\circ}$, with the strain values for (c) and (d) being very close to each other. This order coincides directly to the amount of diffuse thinning within the fracture process zone and thereby, to the fracture surface morphology. The general trend is that the greater the diffuse thinning, the greater the overall strain at the onset of the macroscopic localization. Notice that the results presented in Fig. 2 are representative for all three realizations of random distribution analyzed.

Figures 3(a)-(g) display the distribution of the void volume fraction in seven dif-

\footnotetext{
${ }^{2}$ In this paper, the localization of the plastic flow in a deformation band across the plate cross section is referred to as the macroscopic localization, and the localization in the ligaments connecting neighboring void nucleation sites (leading to void coalescence) is referred to as microscopic localization, see Tekoğlu et al. (2015) for a detailed discussion.
} 
ferent plates loaded until final failure, where for each plate $N_{\mathrm{p}}=10$ and $R_{\mathrm{p}} / L_{\mathrm{e}}=6$, with identical random distribution. The void nucleation sites are circular in the plate displayed in Fig. 3(a) and elliptic for all other plates with the aspect ratio being $R=5$ for (b)-(d) and $R=10$ for (e)-(g). The orientation angle of the void nucleation sites is $\psi=0^{\circ}$ for (b) and (e), $\psi=45^{\circ}$ for (c) and (f), and $\psi=90^{\circ}$ for (d) and (g). Figure 3(h) (respectively Fig. 3(i)) compares the stress-strain curves for the plates displayed in (b)(d) (respectively (e)-(g)) and (a). With an exception for $\psi=0^{\circ}$ (see (b) and (e)), all plates exhibit a slant crack surface morphology with virtually identical shape and location. Nonetheless, the overall strain at the onset of the macroscopic localization clearly correlates with the orientation angle, and is greatest for $\psi=0^{\circ}$, followed in decreasing order by the plates with circular nucleation sites, plates with $\psi=90^{\circ}$, and plates with $\psi=45^{\circ}$. The effect of the aspect ratio on the localization strain is less pronounced, and Figs. 3(h) and (i) reveal that the localization strain is marginally less for $R=5$ relative to $R=10$, when maintaining the orientation angle fixed. The results (not displayed here) for two additional sets of seven plates with $N_{\mathrm{p}}=10$ and $R_{\mathrm{p}} / L_{\mathrm{e}}=6$, and different random distributions, indicated the same trends.

The investigation indicated that plate materials with a high number of large nucleation sites consistently yield slant crack surface morphology, independent of the orientation and aspect ratio. This is demonstrated in Fig. 4 for seven plates, each with $N_{\mathrm{p}}=100$ and $R_{\mathrm{p}} / L_{\mathrm{e}}=9$. In all cases except, the crack slants and material separates at a $45^{\circ}$ angle to the main loading direction, except for (g) where the angle is around $20^{\circ}$. It should be noted that apart from the plates in (d) and (g), the void volume fraction remains virtually uniform throughout the matrix material, except for the narrow localization band where separation occurs. A strong interaction between the local shear bands emanating from the individual nucleation sites causes this narrow band to form and prohibits the evolution of diffuse damage. The plate microstructure for $\psi=90^{\circ}$ (represented by (d) and $(\mathrm{g})$ ), however, allows the formation of several bands where plastic strain and, in turn, void volume fraction concentrates. Thus, several alternative crack paths develop in the course of the loading. The material eventually separates on the energetically most favorable path, which therefore, is not necessarily a straight line (see also discussion in Tekoğlu and Nielsen, 2019). The failure mechanisms 
have a significant influence on the energy dissipation associated with the material separation (see Section 3.2), which is manifested by the limited ductility apparent from the overall stress-strain responses in Figs. 4(h)-(i). Considering the overall strain at the macroscopic localization, the order of the different material configurations is identical to the case $N_{\mathrm{p}}=10-R_{\mathrm{p}} / L_{\mathrm{e}}=6$ (compare Figs. 4(h)-(i)). Two additional sets of seven plates - with different random distributions of nucleation sites - indicated the same trends as in Fig. 4; the only difference being that for certain plates, the crack had a cup-cone-like morphology.

In terms of crack surface morphologies and the macroscopic strain at localization, material configurations with $N_{\mathrm{p}}=25$ represent the intermediate cases between $N_{\mathrm{p}}=10$ and $N_{\mathrm{p}}=100$. The results for seven plates with $N_{\mathrm{p}}=25-R_{\mathrm{p}} / L_{\mathrm{e}}=6$ are displayed in Fig. 5 for $R=5$ and $R=10$. At the two extremes, $N_{\mathrm{p}}=10-R_{\mathrm{p}} / L_{\mathrm{e}}=3$ and $N_{\mathrm{p}}=100-$ $R_{\mathrm{p}} / L_{\mathrm{e}}=9$, the crack surface morphology is either cup-cup or slanted independent of the spatial nucleation site distribution. For the intermediate case of $N_{\mathrm{p}}=25-R_{\mathrm{p}} / L_{\mathrm{e}}=6$, however, the crack morphology is sensitive to the distribution and the surface morphology is inconclusive, signaling a tearing crack is likely to propagate as a mixture of cup-cup, cup-cone, and slanting. This is exemplified in Figs. 5(a)-(g). Nonetheless, the dependency of the macroscopic localization strain on the orientation of the nucleation sites is identical for all tested combinations of $N_{\mathrm{p}}-R_{\mathrm{p}} / L_{\mathrm{e}}$, except for the plates with $N_{\mathrm{p}}=10$ and $R_{\mathrm{p}} / L_{\mathrm{e}}=3$ (see Fig. 2).

It is important to remember that the above findings rest on analyses for a fixed strain hardening exponent for the matrix material $(N=0.10$, see Eq. (1)). The value of $N$ influences the localization and hence, the crack surface morphology as exemplified in Fig. 6. All the material and geometric parameters including the spatial distribution of the void nucleation sites, are the same for the plates in Figs. 6(a) and (b) (respectively, Figs. 6(c) and (d)) except that $N=0.10$ in (a) and $N=0.01$ in (b) (respectively, $N=0.10$ in (c) and $N=0.20$ in (d)). The results indicate that the crack surface morphology shifts from cup-cup to slanted (or in certain cases to cup-cone) for a lesser $N$-value, and from a slanted (or cup-cone) to a cup-cup-like morphology for a greater $N$-value. This conclusion holds for the 12 different sets of plates analyzed, each set containing two plates, with the only difference between them being the matrix strain 
hardening exponent. The results (not presented here) displayed a clear shift in the crack surface morphology for 8 sets of the 12 simulations.

\subsection{Tearing energy of plates with heterogeneous microstructures}

Andersen et al. (2020) demonstrated that microstructural heterogeneity significantly influences the tearing energy of metal plates; however, they limited their attention to circular nucleation sites. In the following, the plate tearing energy is evaluated from the new set of results presented in Sections 3.1, including elliptic nucleation sites. The approach for evaluating the energy follows that of Nielsen and Hutchinson (2012), where the load-deflection curves are consulted and the energy of the tearing event is related to the deformation after the onset of local plate thinning (i.e., beyond the peak load, see Fig. 1(d)). The level of the peak load (evaluated where $\Delta H / H_{0}=N$ ) is essentially not influenced by the heterogeneous material configuration (see also the discussion in Andersen et al., 2020) and is considered the starting point for the local plate thinning (see Fig. 1). The tearing energy is thereby identified as the energy under the load-deflection curve from the onset of thinning at $\Delta H / H_{0}=N$ to the complete loss of load-carrying capacity (see Fig. 1).

Figure 7 presents the tearing energy for heterogeneous plates with circular void nucleation sites of size $R_{\mathrm{p}} / L_{\mathrm{e}}=3,6$, and 9 , randomly distributed in the metal matrix with a strain hardening exponent of $N=0.1$. By increasing the size of the nucleation sites while the void nucleation parameters remain constant, the porosity of the plate naturally increases, resulting in the lowest tearing energy for the plate containing the largest nucleation sites. Similarly, the tearing energy decreases when increasing the number of nucleation sites. The lower tearing energy relates both to an increase in the volume fraction of damage that potentially can nucleate and to the increased intensity of interaction between the nucleated voids. Three realizations of the nucleation site distributions yield a statistical variation of the void interaction mechanisms resulting in an interval of confidence ( $3 \sigma$ with $\sigma$ being the standard deviation) that is the largest when the crack surface morphology is inconclusive (see Fig. 7).

Figure 8 depicts the tearing energy as a function of the number of nucleation sites, $N_{\mathrm{p}}$, for the intermediate size, $R_{\mathrm{p}} / L_{\mathrm{e}}=6$, and void aspect ratios of $R=5$ and $R=10$ (respectively, in Figs. 8(a) and (b)). Here, the results for circular nucleation sites as 
reference are included. For fixed orientation, the increase in the number of nucleation sites follows the trends found for the circular sites. As the number and thereby, the volume fraction of void nucleation sites increases, the tearing energy decreases, revealing a significant effect from the nucleation site orientation. The tearing energy is the greatest when the primary axis of the nucleation site is parallel to the main loading direction $\left(\psi=0^{\circ}\right)$; it actually exceeds that of the corresponding circular sites. Recall that the realization of the distribution and volume fraction are identical in these simulations. The tearing energy, however, displays a decrease when rotating the nucleation sites to $\psi=45^{\circ}$, and only regains a marginally greater level when considering a $90^{\circ}$ orientation (minor axis along the loading direction). Moreover, the effect of the nucleation site orientation is amplified when the aspect ratio is increased (compare Figs. 8 (a) and (b)). The mechanism behind this is that as the aspect ratio increases for a $0^{\circ}$ orientation of the nucleation sites, the intervoid ligaments between the nucleation sites increase in the through-thickness direction of the plate, inhibiting the interaction of the nucleating sites such that profound thinning of the plate must occur before failure (vice versa for a $90^{\circ}$ orientation). At an orientation of $\psi=45^{\circ}$, the nucleation sites approximately align with the orientation of the macroscopic shear band related to the slant failure, reducing the plate thinning and tearing energy substantially.

The orientation effect is also evident from Fig. 9, indicating the tearing energy as a function of the orientation angle, $\psi$, for three different numbers of void nucleation sites $\left(N_{\mathrm{p}}=\{10,25,100\}\right)$. Regardless of the number of void nucleation sites, the tearing energy exhibits a significant decrease when shifting the orientation from $\psi=0^{\circ}$ to $\psi=45^{\circ}$; the energy thereafter increases at $\psi=90^{\circ}$. This non-monotonic dependency between the tearing energy and the influence of the orientation angle is most distinct for the highest aspect ratio considered $(R=10)$.

Both the aspect ratio and orientation of the nucleation sites significantly alter the tearing energy, independent of the size of the nucleation sites. Fig. 10 presents the variation in tearing energy when the aspect ratio increases in the interval of $R \in[1,10]$ for three different orientations $\left(\psi=\left\{0^{\circ}, 45^{\circ}, 90^{\circ}\right\}\right)$, and two numbers of nucleation sites $\left(N_{\mathrm{p}}=\{10,100\}\right)$. The influence of the orientation is particularly important when the number of nucleation sites is small (with $N_{\mathrm{p}}=10$ ), and a substantial change to the 
tearing energy is found in the interval $\psi=0^{\circ}$ to $\psi=45^{\circ}$, after which the effect levels off for $\psi=90^{\circ}$. Conversely, the orientation effect is more gradual for a large number of nucleation sites. The trend in these results are independent of the size of the nucleation sites (compare Figs. 10 (a) and (b)) although the level of the tearing energy is different. The energy decreases when increasing the size of the nucleation sites for all aspect ratios considered when the orientation of the void nucleation sites is different from $\psi=0^{\circ}$. Conversely, the energy increases when the major axes of the nucleation sites are aligned with the applied load.

\section{Discussion}

The results of this study confirm that ductile plate tearing occurs through three consecutive stages of localized deformation, with plastic flow being constrained at a reduced length scale in each successive stage. Figures 11(a)-(c) depict these three localization stages for a plate having circular void nucleation sites with $N_{\mathrm{p}}=10-R_{\mathrm{p}} / L_{\mathrm{e}}=3$, and (d) the final separation of the plate. The corresponding curve of the engineering stress versus engineering strain is plotted in Fig. 11(e), where the asterisk $\left(^{*}\right)$ symbols indicate the deformation stages depicted in Figs. 11(a)-(d). The first stage of localized plastic deformation begins with the onset of diffuse thinning in the fracture process zone, see Fig. 11(a). In this stage, plastic deformation is confined in the fracture process zone, and the outer regions of the plate above and below the fracture process zone (see the schematic in Fig.1(a)) unload elastically. As the thinning develops in the fracture process, the porosity increases in the void nucleation sites in this region. At point (b) of the loading history, the plastic flow suddenly localizes into a band inside the thinning region, and porosity ceases to increase in the void nucleation sites outside this band (see Figs. 11(b) and (e)). After the onset of this second localization, which is referred to as "macroscopic localization", the regions off the thinning region within the fracture process zone begin to unload elastically, along with the non-porous parts of the plate that started unloading at point (a). In fact, with the onset of each successive stage of localization, the plastic deformation is confined in a smaller region whereas the unloading extends into a larger region. This can be visualized by comparing Figs. 11(b) and (c): the nucleation sites outside the thinning region hardly evolve 
between the two deformation stages in (b) and (c). Upon further loading, the voids grow inside the macroscopic band of localization, and move closer to each other, until their independent growth is interrupted by the onset of the third localization at point (c), referred to as "microscopic localization". In this stage, the adjacent nucleation sites merge through either void sheeting (as is the case for Fig. 11(c)) or internal necking (for a detailed explanation of the interaction mechanisms between void nucleation sites, see Tekoğlu and Nielsen, 2019). Hereafter, the accelerated void growth is accompanied by a precipitous decrease in the load-carrying capacity of the plate until its final failure (see Fig. 11(d)). Figs. 12(a)-(c) show companion results for a plate with $N_{\mathrm{p}}=100$, $R_{\mathrm{p}} / L_{\mathrm{e}}=9, R=10$, and $\psi=45^{\circ}$. For such plates containing a large number of large particles, the three localized deformation stages are virtually coincident, leading to a slanted crack surface morphology.

Figures 1(a) and (b) illustrate the four stages of ductile plate tearing (the last one being the final failure), respectively, for cup-cup crack propagation as in Fig. 11, and for slanted crack propagation as in Fig. 12. If the overall hardening capacity of the plate is large, crack propagation occurs as described in Fig. 1(a), whereas it propagates as in Fig. 1(b) for low overall hardening capacity. With decreasing overall hardening capacity, the onsets of the three localized deformation stages approach each other, whereas the crack profile tends to assume a slanted form. If the overall hardening capacity decreases to a level where stages (2) and (3) occur (virtually) simultaneously, the crack profile becomes clearly slanted, even if stage (1) occurs earlier, allowing local thinning, as is the case in Figs. 3 (c) and (d). The main finding of this paper (see also Tekoğlu and Nielsen, 2019; Andersen et al., 2020) is that the overall hardening capacity of a plate is determined not only by the strain hardening exponent of the matrix material, but also by the layout of the second phase particles (acting as void nucleation sites) embedded in the matrix material. In stage (1) (when the thinning develops), irrespective of the microstructure and mechanical properties of a ductile plate, two local shear bands emanate from each void nucleation site as soon as the void volume fraction in the nucleation site attains approximately $f=0.1$. One of the two local shear bands is aligned at $+45^{\circ}$ and the other at $-45^{\circ}$ with respect to the tensile direction. Figures 13(a) to (d) exemplify the local shear bands on adjacent 
nucleation sites for four different plates having the same microstructural and mechanical properties, except that the nucleation sites are circular in (a), and have an aspect ratioorientation angle combination of $R=3-\psi=0^{\circ}$ in (b), $R=3-\psi=45^{\circ}$ in (c), and $R=3-\psi=90^{\circ}$ in $(\mathrm{d})$. The crack profiles for these plates are displayed in Figs. 2(a)(d). If the local shear bands that spread out from the individual nucleation sites can accumulate to form a macroscopic shear band spanning the entire plate thickness, multiple voids simultaneously interact and result in a slanted crack, see Figs. 13(c) and $(\mathrm{d})$. If not, the crack propagates by void-by-void interaction, that is, through the merging of adjacent void nucleation sites either via void sheeting or internal necking, resulting in a cup-cup crack morphology, see Figs. 13(a) and (b). Moreover, the smaller the perpendicular distance between void nucleation sites, the easier and earlier they interact. Regarding the perpendicular distance between the closest edges of adjacent nucleation sites, the four plates can be arranged in descending order as (b), (a), (c), and $(\mathrm{d})$. This explains why the tearing energy is less for circular plates than for plates with $\psi=0^{\circ}$. The situation is more complicated for plates with $\psi=45^{\circ}$ and $\psi=90^{\circ}$. For the same spatial distribution, although the distance between the nucleation sites is smaller for plates with $\psi=90^{\circ}$, it is easier for a macroscopic shear band to develop in plates with $\psi=45^{\circ}$. This explains the non-monotonic dependency of the tearing energy on the orientation angle given in Fig. 9. The interaction between voids is also easier for a smaller strain hardening exponent of the matrix material, as evidenced from the crack surface morphologies displayed in Fig. 6.

A word of caution is necessary. Throughout this work (also in Tekoğlu and Nielsen, 2019; Andersen et al., 2020), a strain-based void nucleation law is employed, which is an acceptable representation of reality only if the void nucleation occurs through interface decohesion. If void nucleation is due to particle fracture, however, a stressbased nucleation law is more appropriate. Moreover, it is well known that the critical strain (or stress) for void nucleation depends on the size, shape, and orientation of the particles (see e.g. Lassance et al., 2007; Tekoğlu and Pardoen, 2010). Nevertheless, the presented framework successfully captures the qualitative effect of the heterogeneous microstructure on the plate tearing mechanism and energy. 


\section{Conclusions}

The key findings of this study are as follows.

- The crack surface morphology in ductile plate tearing is related to the overall hardening capacity of the plate, which is determined not only by the strain hardening exponent of the matrix material, but also by the spatial distribution, size, shape, and orientation of the second phase particles embedded in the matrix.

- The tearing energy increases with the overall hardening capacity, and for sufficiently large matrix strain hardening, the crack shifts to propagate by void-byvoid interaction, leading to a cup-cup crack surface morphology.

- For a plate with sufficiently low overall hardening capacity, the tearing energy is low and the crack propagates by simultaneous interaction between multiple voids captured in a macroscopic shear band, leading to a slanted crack surface morphology.

\section{Acknowledgments}

The authors gratefully acknowledge the financial support by TÜBİTAK (Project No: 315M133). RGA is financially supported by the Department of Mechanical Engineering at the Technical University of Denmark in the project "Advancing Numerical Analysis of Large Scale Crack Propagation in Plate Structures". KLN is financially supported by Independent Research Fund Denmark, partly, in the project "Advanced Damage Models with InTrinsic Size Effects" (Grant no: DFF-7017-00121), and, partly, in the project "Why, Where and When metals fail" (Grant no: 0136-00194B).

\section{References}

Andersen, R., Felter, C.L., Nielsen, K.L., 2019. Micro-mechanics based cohesive zone modeling of full scale ductile plate tearing: From initiation to steady-state. International Journal of Solids and Structures 160, 265-275.

Andersen, R., Tekoğlu, C., Nielsen, K., 2020. Cohesive traction-separation relations for tearing of ductile plates with randomly distributed void nucleation sites. Int. J. Fracture 224, 1-12. doi:10.1007/s10704-020-00454-2. 
Asserin-Lebert, A., Besson, J., Gourgues, A.F., 2005. Fracture of 6056 aluminum sheet materials: effect of specimen thickness and hardening behavior on strain localization and toughness. Materials Science and Engineering: A 395, 186 - 194.

Bron, F., Besson, J., Pineau, A., 2004. Ductile rupture in thin sheets of two grades of 2024 aluminum alloy. Materials Science and Engineering: A 380, 356 - 364.

Chu, C., Needleman, A., 1980. Void nucleation effects in biaxially stretched sheets. J. Eng. Mater. Technol. 102, 249-256.

Dassault Systèmes Simulia Corp., 2016. ABAQUS version 2016 documentation.

Cox, T.B., Low, J.R., 1974. An investigation of the plastic fracture of AISI 4340 and 18 Nickel-200 grade maraging steels. Metall. Trans. 5, 1457-1470.

Dighe, M.D., Gokhale, A.M., Horstemeyer, M.F., 2002. Second phase cracking and debonding observations in the fatigue damage evolution of a cast $\mathrm{Al}-\mathrm{Si}-\mathrm{Mg}$ alloy. Metall. Mater. Trans 33A, $1-8$.

Gurland, J., 1972. Observations on the fracture of cementite particles in a spheroidized 1.05\% C steel deformed at room temperature. Acta Metallurgica 20, 735 - 741.

Gurson, A., 1977. Continuum theory of ductile rupture by void nucleation and growth - part I: yield criteria and flow rules for porous ductile media. ASME J. Eng. Mater. Technol. 99, 2-15.

Hickey, W., Ravi-Chandar, K., 2015. Transition from flat to slant fracture in ductile materials. Recent Trends in Fracture and Damage Mechanics , 215-235.

Lassance, D., Fabrègue, D., Delannay, F., Pardoen, T., 2007. Micromechanics of room and high temperature fracture in 6xxx al alloys. Progress in Materials Science 52, $62-129$.

Lewandowski, J.J., Liu, C., Hunt, W.H., 1989. Effects of matrix microstructure and particle distribution on fracture of an aluminum metal matrix composite. Materials Science and Engineering: A 107, $241-255$. 
Marino, B., Mudry, F., Pineau, A., 1985. Experimental study of cavity growth in ductile rupture. Engineering Fracture Mechanics 22, 989 - 996.

Nielsen, K., Hutchinson, J., 2012. Cohesive traction-separation laws for tearing of ductile metal plates. Int. J. Imp. Eng. 48, 15-23.

Pardoen, T., Hachez, F., Marchioni, B., Blyth, P., Atkins, A., 2004. Mode i fracture of sheet metal. J. Mech. Phys. Solids 52, $423-452$.

Pineau, A., Benzerga, A., Pardoen, T., 2016. Failure of metals I: Brittle and Ductile Fracture. Acta Materialia 107, 424-483.

Srivastava, A., Osovski, S., Needleman, A., 2017. Engineering the crack path by controlling the microstructure. J. Mech. Phys. Solids 100, $1-20$.

Srivastava, A., Ponson, L., Osovski, S., Bouchaud, E., Tvergaard, V., Needleman, A., 2014. Effect of inclusion density on ductile fracture toughness and roughness. J. Mech. Phys. Solids 63, $62-79$.

Tekoğlu, C., Hutchinson, J., Pardoen, T., 2015. On localization and void coalescence as a precursor to ductile fracture. Phil. Trans. R. Soc. A373.

Tekoğlu, C., Nielsen, K., 2019. Effect of damage-related microstructural parameters on plate tearing at steady state. European J. Mech. A/Solids 77, 103818.

Tekoğlu, C., Pardoen, T., 2010. A micromechanics based damage model for composite materials. International Journal of Plasticity 26, 549 - 569.

Tvergaard, V., 1981. Influence of voids on shear band instabilities under plane strain condition. Int. J. Fract. Mech. 17, 389-407.

Tvergaard, V., Hutchinson, J., 2002. Two mechanisms of ductile fracture: Void by void growth versus multiple void interaction. Int. J. Solid and Struct. 39, 3581-3597.

Tvergaard, V., Needleman, A., 1984. Analysis of the cup-cone fracture in a round tensile bar. Acta Metallurgica 32, 157-169. 


\section{Figures}

(a)<smiles>CC(C)C</smiles>

(c)

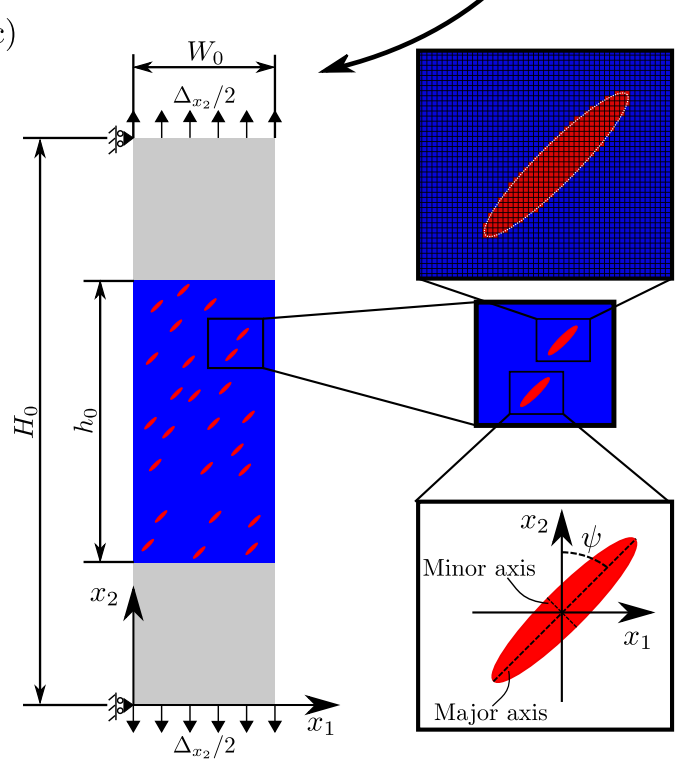

(b)

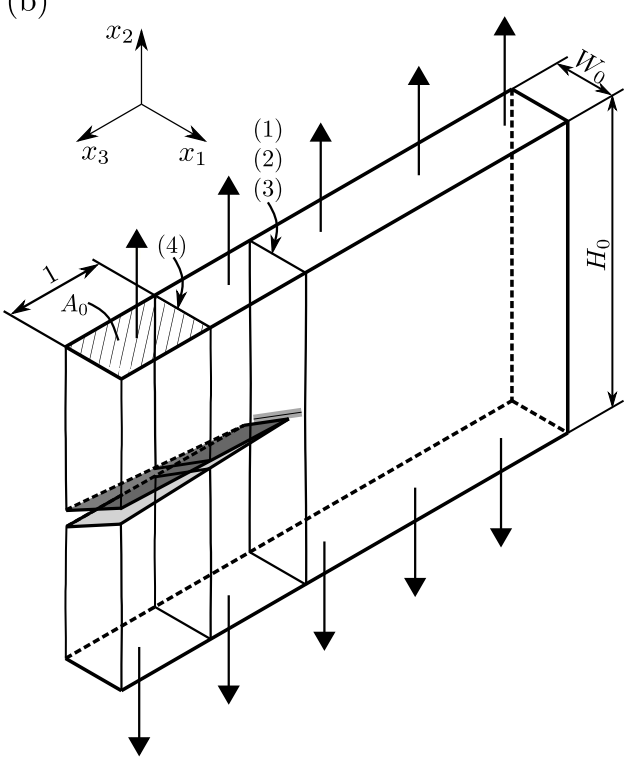

(d)

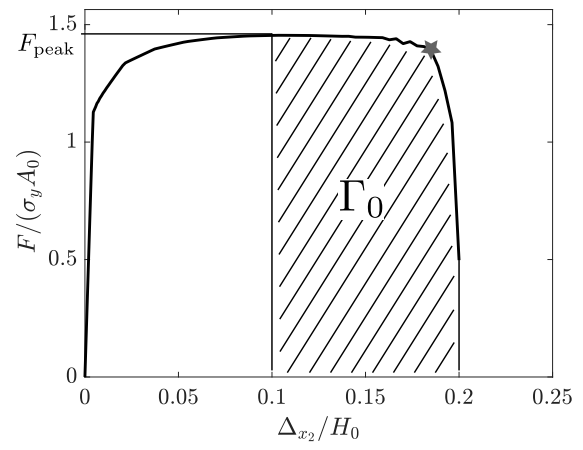

Figure 1: Illustration of large-scale mode I plate tearing and the 2D plane strain cross sections located in front of the crack tip for (a) cup-cup crack propagation and (b) slanted crack propagation. (c) Schematic of the cross section of a plate containing randomly distributed, elliptic void nucleation sites oriented at $45^{\circ}$ with respect to the main loading direction, within the fracture process zone (middle section with a height of $h_{0}$ ). The height of the plate is considered to be four times its width, $H_{0}=4 W_{0}$, and the height of fracture process zone is $h_{0}=2 W_{0}$. The prescribed displacement boundary conditions and the meshing of a void nucleation zone are as illustrated. (d) The load-deflection response curve defining the peak force, $F_{\text {peak }}$, at the onset of local plate thinning, and the corresponding tearing energy, $\Gamma_{0}$. 


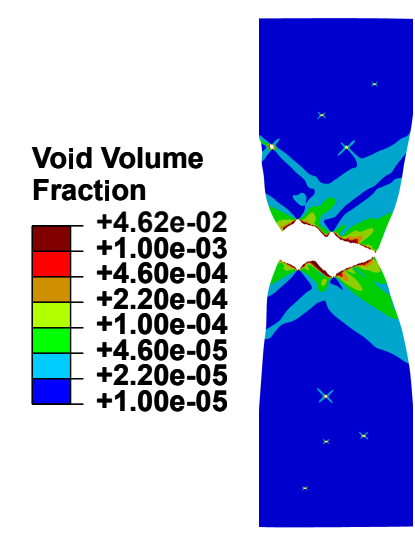

(a) Circular

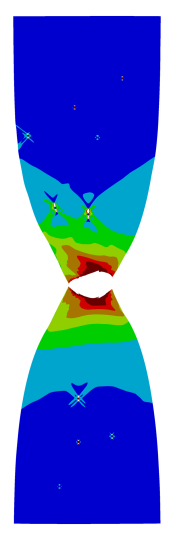

(b) $R=3, \psi=0^{\circ}$

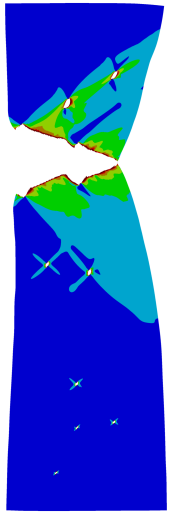

(c) $R=3, \psi=45^{\circ}$

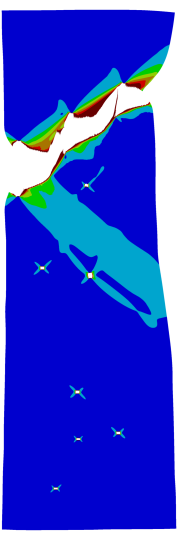

(d) $R=3, \psi=90^{\circ}$

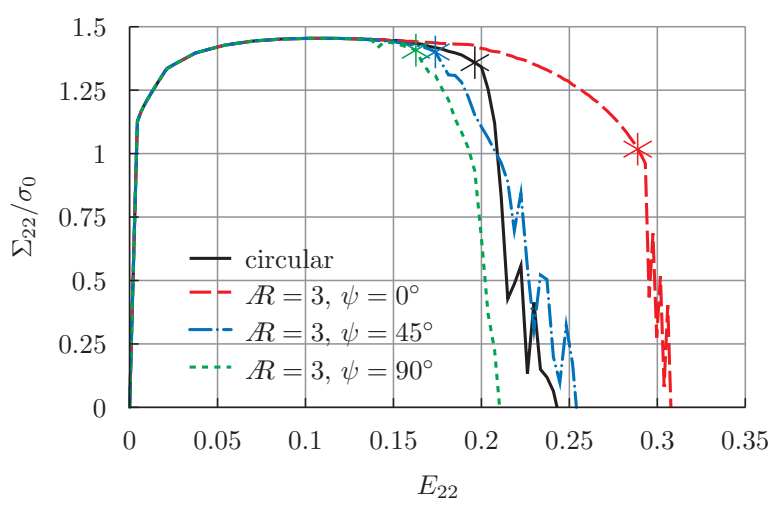

(e)

Figure 2: $(\mathrm{a}-\mathrm{d})$ Developed void volume fraction at the final failure in the fracture process zone for four plates with $N_{\mathrm{p}}=10-R_{\mathrm{p}} / L_{e}=3$. The material and geometric parameters of the four plates, including the spatial distribution of the centers of the void nucleation sites are the same, except for the aspect ratio $(R)$ and orientation angle $(\psi)$ of the nucleation sites: (a) circular, (b) $R=3-\psi=0^{\circ}$, (c) $R=3-\psi=45^{\circ}$, and (d) $R=3-\psi=90^{\circ}$. (e) Variation of the normalized engineering stress $\Sigma_{22} / \sigma_{0}$ with the engineering strain $E_{22}$ for the four plates. 


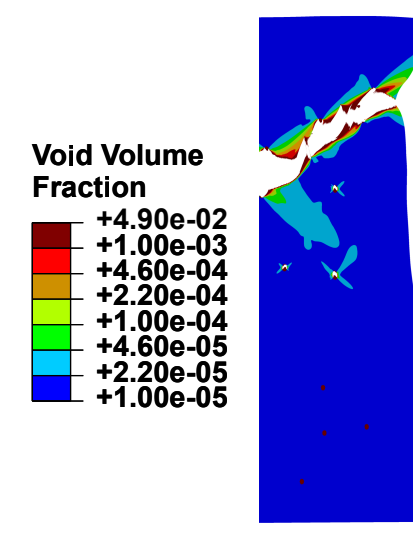

(a) circular

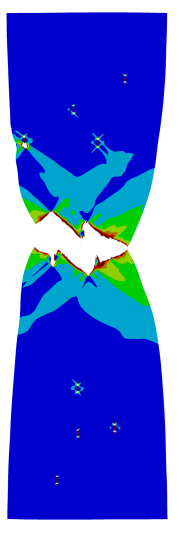

(b) $R=5, \psi=0^{\circ}$

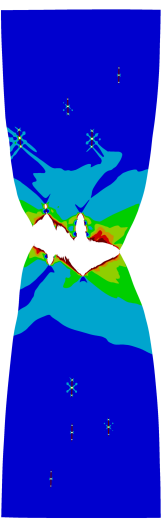

(e) $R=10, \psi=0^{\circ}$

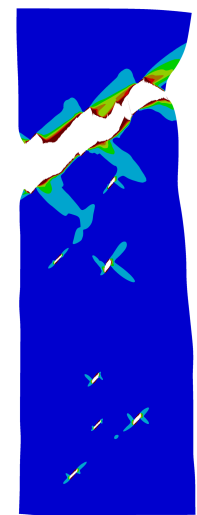

(c) $R=5, \psi=45^{\circ}$

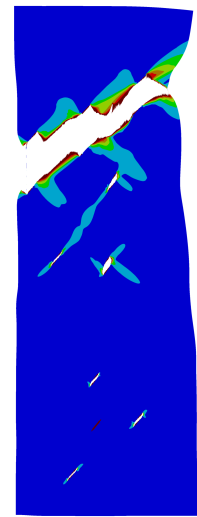

(f) $R=10, \psi=45^{\circ}$

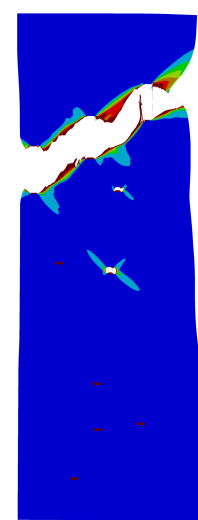

(d) $R=5, \psi=90^{\circ}$

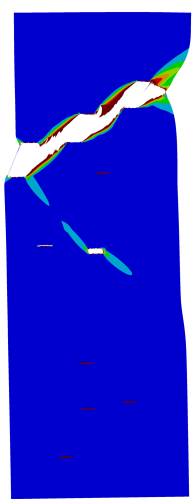

(g) $R=10, \psi=90^{\circ}$

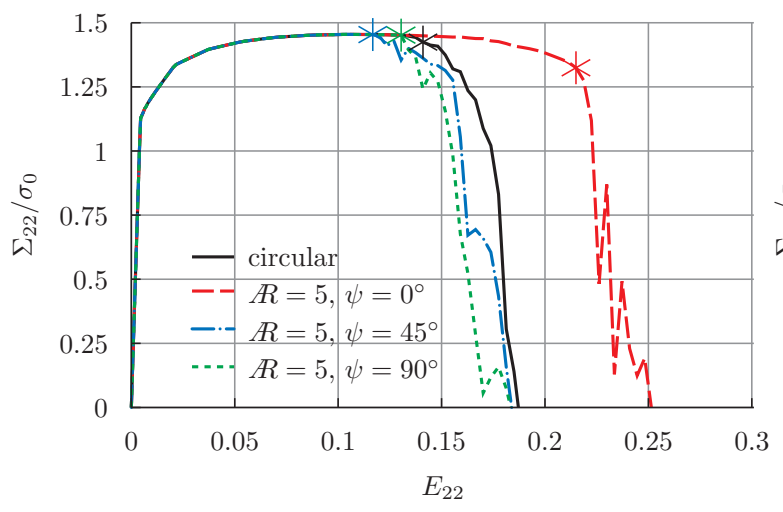

(h)

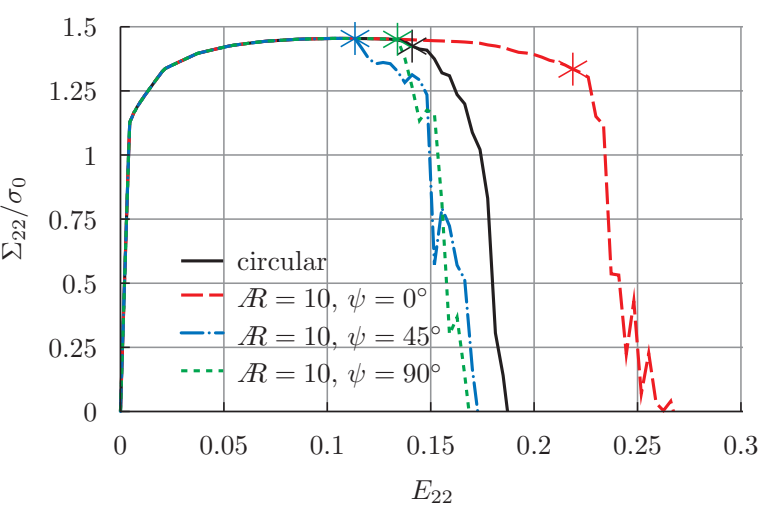

(i)

Figure 3: $(\mathrm{a}-\mathrm{g})$ Developed void volume fraction at the final failure in the fracture process zone for seven plates with $N_{\mathrm{p}}=10-R_{\mathrm{p}} / L_{e}=6$. The material and geometric parameters of the seven plates, including the spatial distribution of the centers of the void nucleation sites are the same, except for the aspect ratio $(R)$ and orientation angle $(\psi)$ of the nucleation sites: (a) circular, (b) $R=5-\psi=0^{\circ}$, (c) $R=5-\psi=45^{\circ}$, (d) $R=5-\psi=90^{\circ}$, (e) $R=10-\psi=0^{\circ}$, (f) $R=10-\psi=45^{\circ}$, and (g) $R=10-\psi=90^{\circ}$. Variation of the normalized engineering stress $\Sigma_{22} / \sigma_{0}$ with the engineering strain $E_{22}$ for the plates: (h) displayed in (a) and (b-d), (i) displayed in (a) and (e-g). 


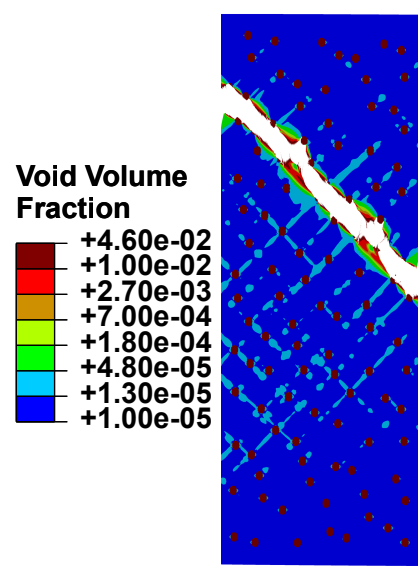

(a) circular

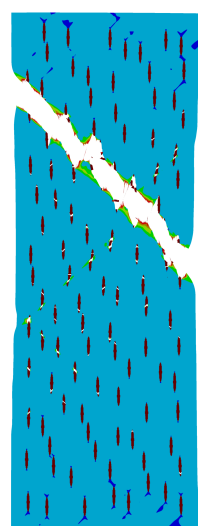

(b) $R=5, \psi=0^{\circ}$

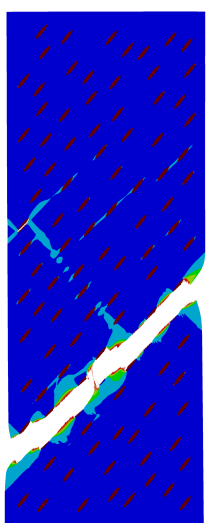

(c) $R=5, \psi=45^{\circ}$

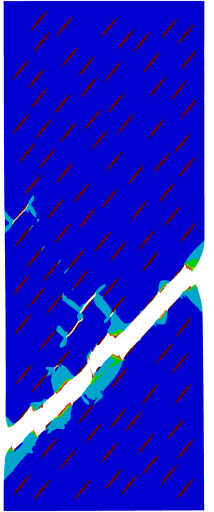

(f) $R=10, \psi=45^{\circ}$

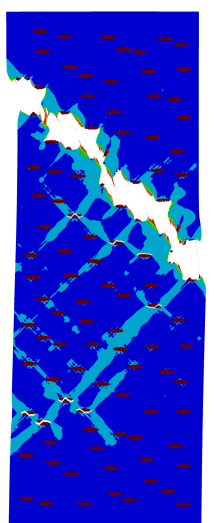

(d) $R=5, \psi=90^{\circ}$

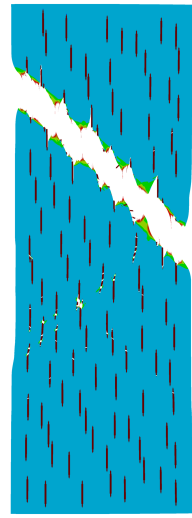

(e) $R=10, \psi=0^{\circ}$ (g) $R=10, \psi=90^{\circ}$

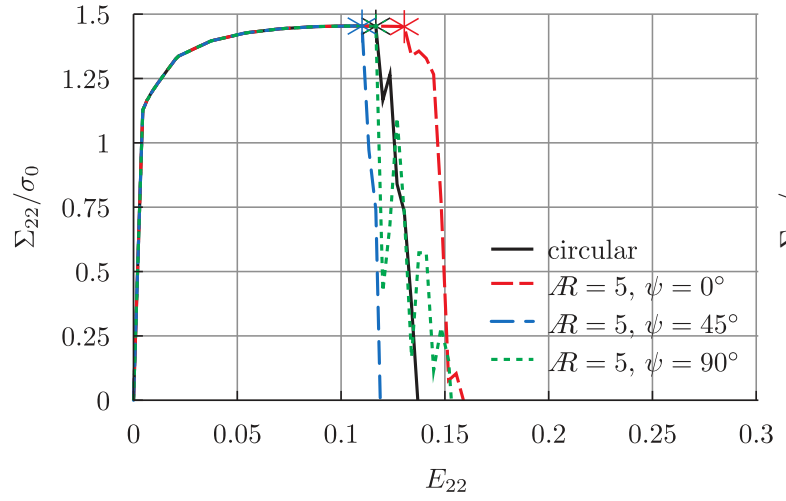

(h)

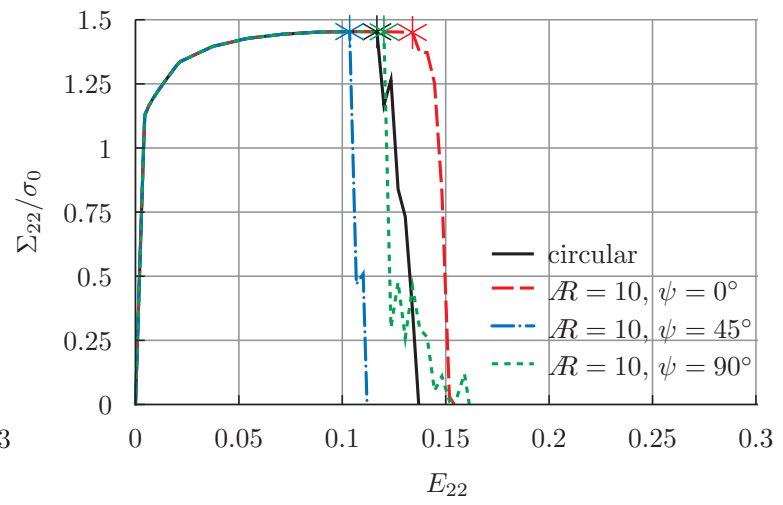

(i)

Figure 4: $(\mathrm{a}-\mathrm{g})$ Developed void volume fraction at the final failure in the fracture process zone for seven plates with $N_{\mathrm{p}}=100-R_{\mathrm{p}} / L_{e}=9$. The material and geometric parameters of the seven plates, including the spatial distribution of the centers of the void nucleation sites are the same, except for the aspect ratio $(R)$ and orientation angle $(\psi)$ of the nucleation sites: (a) circular, (b) $R=5-\psi=0^{\circ}$, (c) $R=5-\psi=45^{\circ}$, (d) $R=5-\psi=90^{\circ}$, (e) $R=10-\psi=0^{\circ}$, (f) $R=10-\psi=45^{\circ}$, and (g) $R=10-\psi=90^{\circ}$. Variation of the normalized engineering stress $\Sigma_{22} / \sigma_{0}$ with the engineering strain $E_{22}$ for the plates: (h) displayed in (a) and (b-d), (i) displayed in (a) and (e-g). 


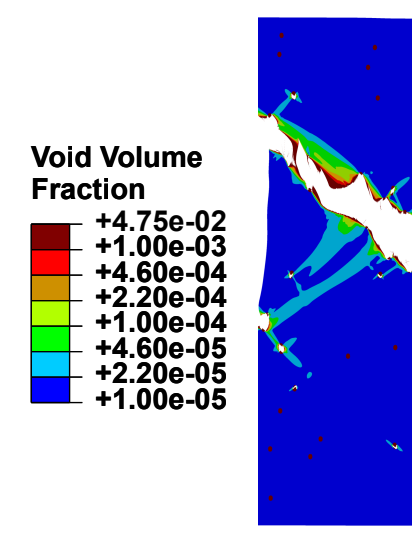

(a) circular

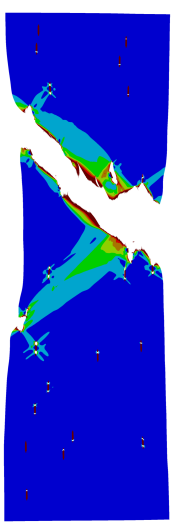

(b) $R=5, \psi=0^{\circ}$

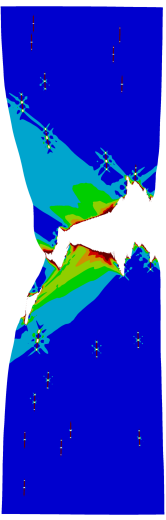

(e) $R=10, \psi=0^{\circ}$

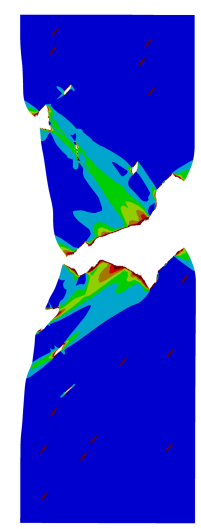

(c) $R=5, \psi=45^{\circ}$

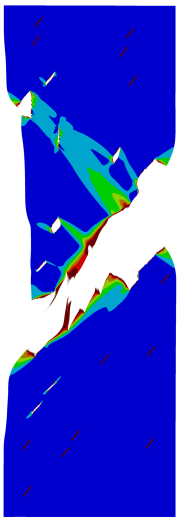

(f) $R=10, \psi=45^{\circ}$

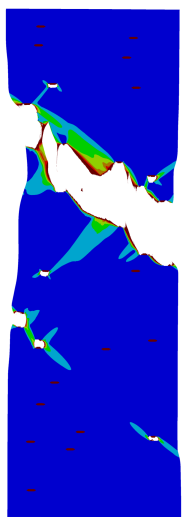

(d) $R=5, \psi=90^{\circ}$

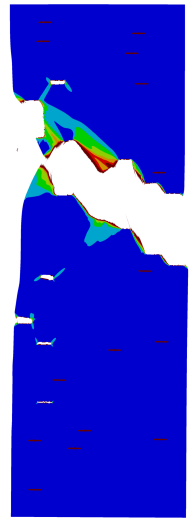

(g) $R=10, \psi=90^{\circ}$

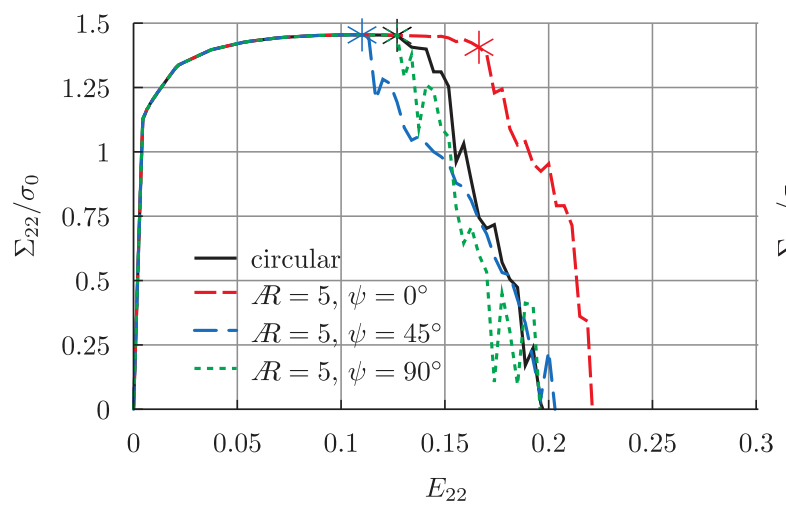

(h)

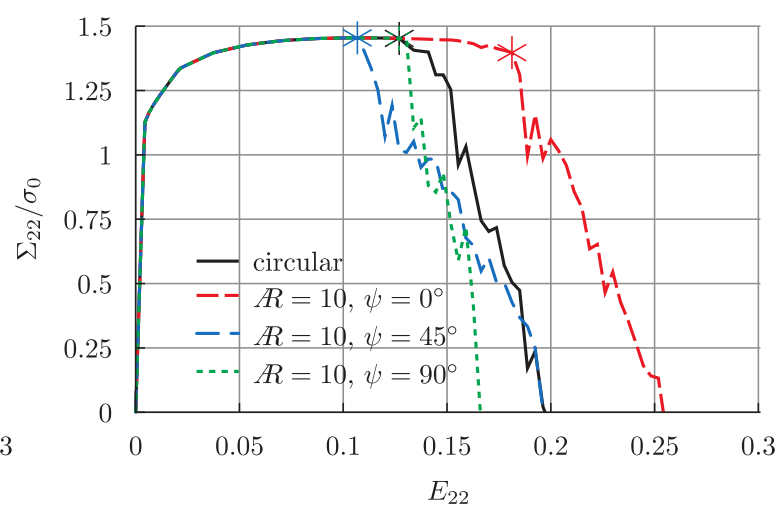

(i)

Figure 5: $(\mathrm{a}-\mathrm{g})$ Developed void volume fraction at the final failure in the fracture process zone for seven plates with $N_{\mathrm{p}}=25-R_{\mathrm{p}} / L_{e}=6$. The material and geometric parameters of the seven plates, including the spatial distribution of the centers of the void nucleation sites are the same, except for the aspect ratio $(R)$ and orientation angle $(\psi)$ of the nucleation sites: (a) circular, (b) $R=5-\psi=0^{\circ}$, (c) $R=5-\psi=45^{\circ}$, (d) $R=5-\psi=90^{\circ}$, (e) $R=10-\psi=0^{\circ}$, (f) $R=10-\psi=45^{\circ}$, (g) $R=10-\psi=90^{\circ}$. Variation of the normalized engineering stress $\Sigma_{22} / \sigma_{0}$ with the engineering strain $E_{22}$ for the plates: (h) displayed in (a) and (b-d), (i) displayed in (a) and (e-g). 


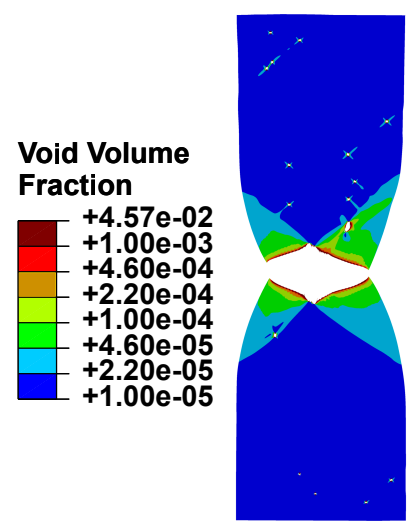

(a) $N_{\mathrm{p}}=18, N=0.10$

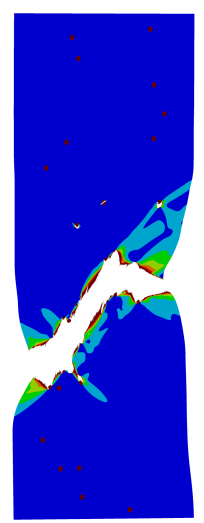

(c) $N_{\mathrm{p}}=25, N=0.10$

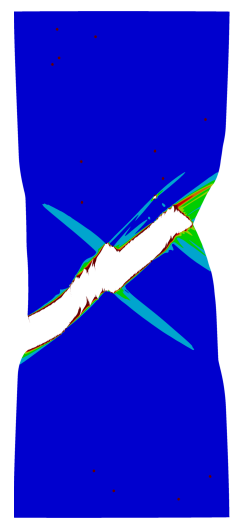

(b) $N_{\mathrm{p}}=18, N=0.01$

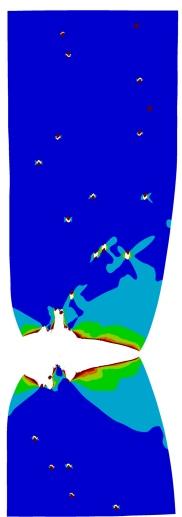

(d) $N_{\mathrm{p}}=25, N=0.20$

Figure 6: (a-d) Developed void volume fraction at the final failure in the fracture process zone for four plates with circular void nucleation sites. The material and geometric parameters including the spatial distribution of the void nucleation sites, are the same for $(a, b)$ (respectively, $(c, d)$ ) except that $N=0.10$ in (a) and $N=0.01$ in (b) (respectively, $N=0.10$ in (c) and $N=0.20$ in (d)). For (a,b) $N_{\mathrm{p}}=18, R_{\mathrm{p}} / L_{e}=3$, and for $(\mathrm{c}, \mathrm{d}) N_{\mathrm{p}}=25, R_{\mathrm{p}} / L_{e}=6$. 


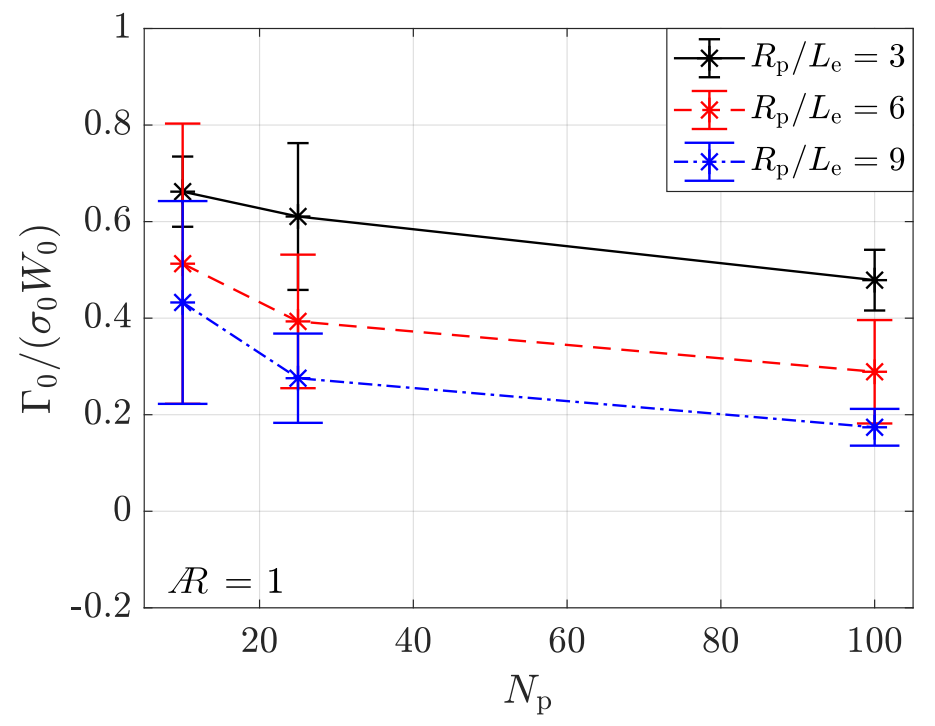

Figure 7: Tearing energy, $\Gamma_{0}$, as a function of the number of spherical $(R=1)$ void nucleation sites, $N_{\mathrm{p}}$, for three different sizes $\left(R_{\mathrm{p}} / L_{e}=\{3,6,9\}\right)$. The mean value and interval of confidence $(3 \sigma$, with $\sigma$ being the standard deviation), are indicated. 


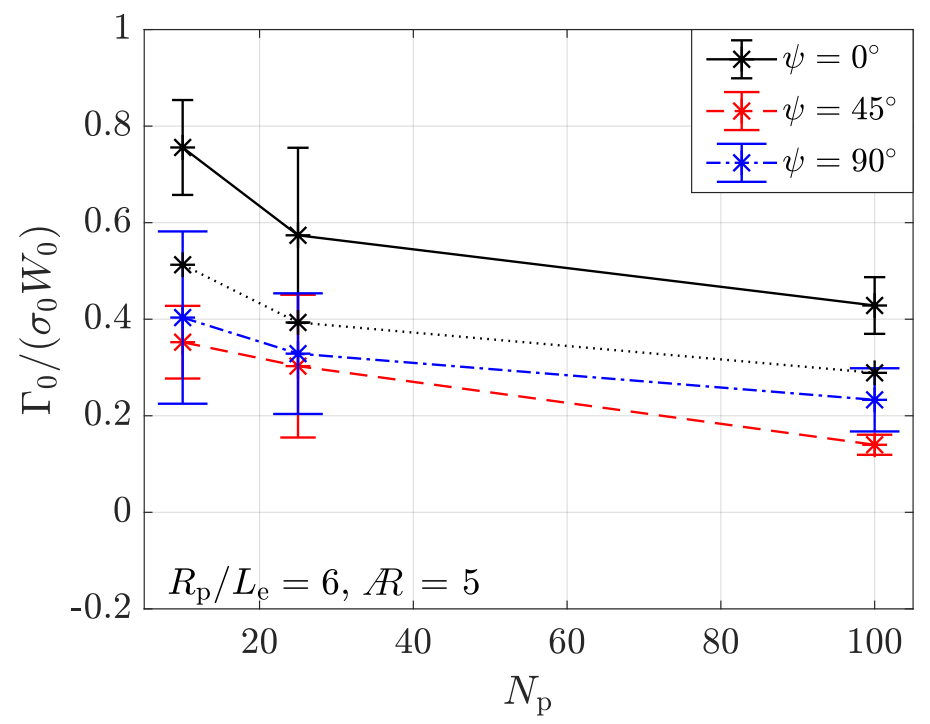

(a)

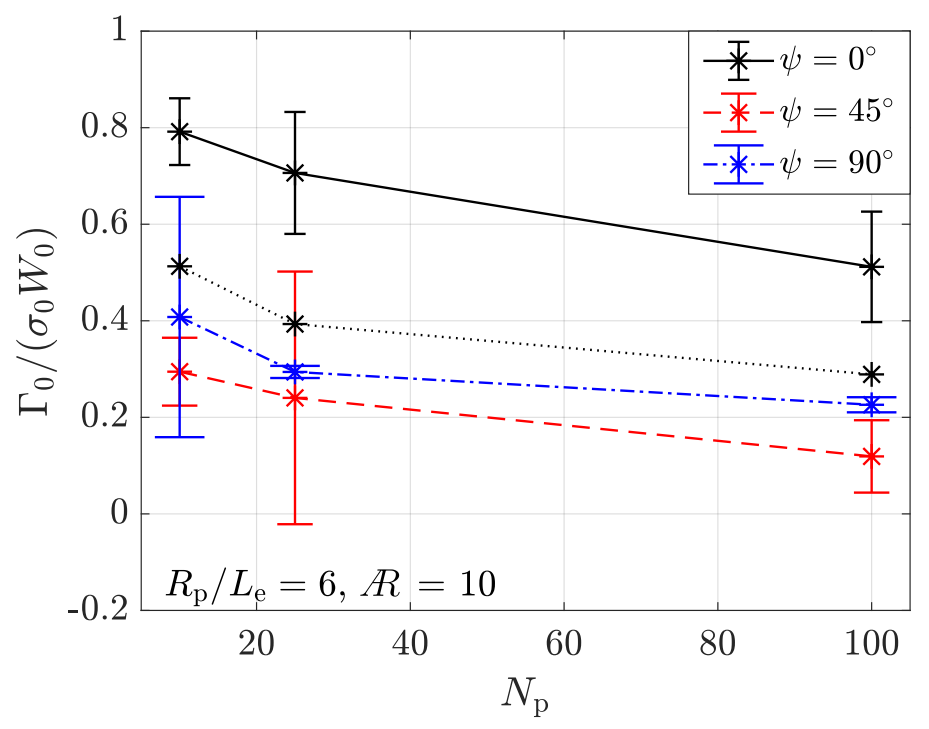

(b)

Figure 8: Tearing energy, $\Gamma_{0}$, as a function of the number of void nucleation sites, $N_{\mathrm{p}}$, for a size of $R_{\mathrm{p}} / L_{e}=6$ and aspect ratio of (a) $R=5$ and (b) $R=10$. The mean value and interval of confidence ( $3 \sigma$, with $\sigma$ being the standard deviation), are indicated for three different orientations $\left(\psi=\left\{0^{\circ}, 45^{\circ}, 90^{\circ}\right\}\right)$. 


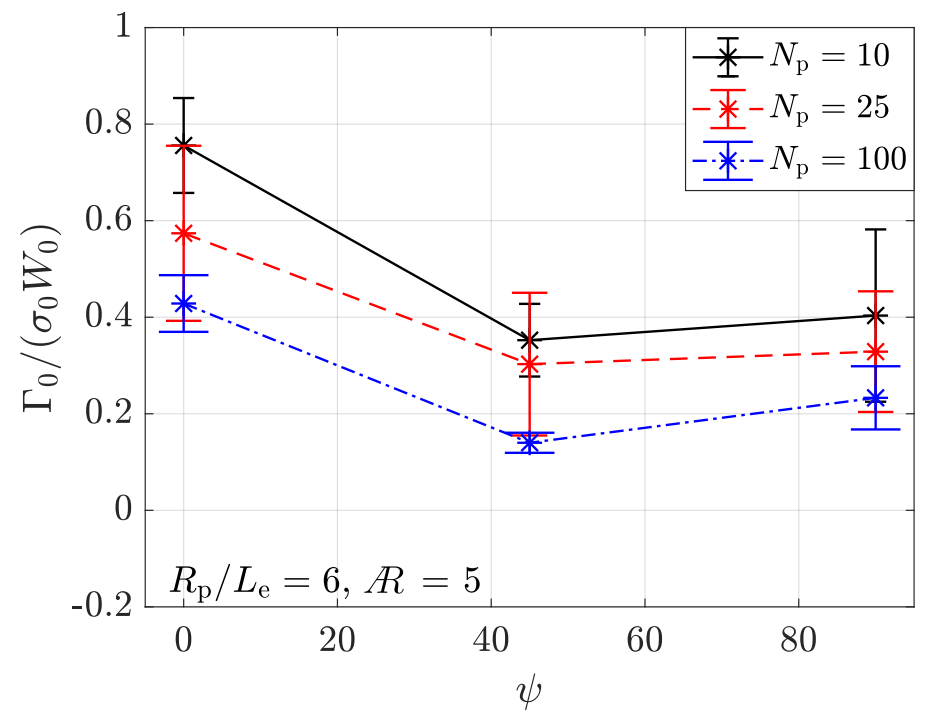

(a)

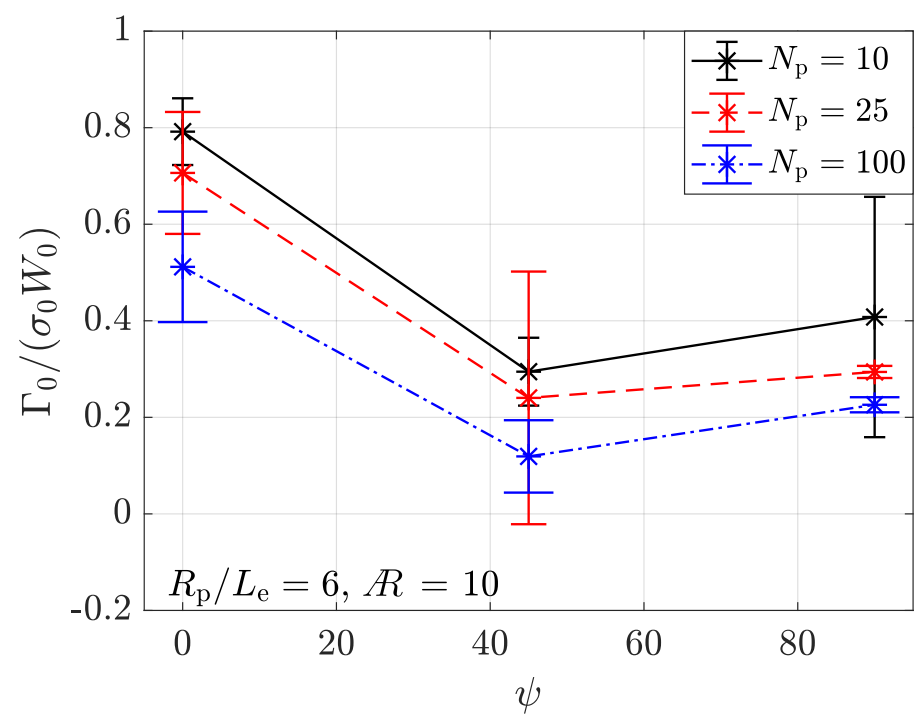

(b)

Figure 9: Tearing energy, $\Gamma_{0}$, as a function of the orientation of the void nucleation sites, $\psi$, for an aspect ratio of (a) $R=5$ and (b) $R=10$. The void nucleation sites are of size $R_{\mathrm{p}} / L_{e}=6$ and the number of nucleation sites varies between $N_{\mathrm{p}}=\{10,25,100\}$. Results are displayed for the mean value and interval of confidence of $3 \sigma$ with $\sigma$ being the standard deviation. 


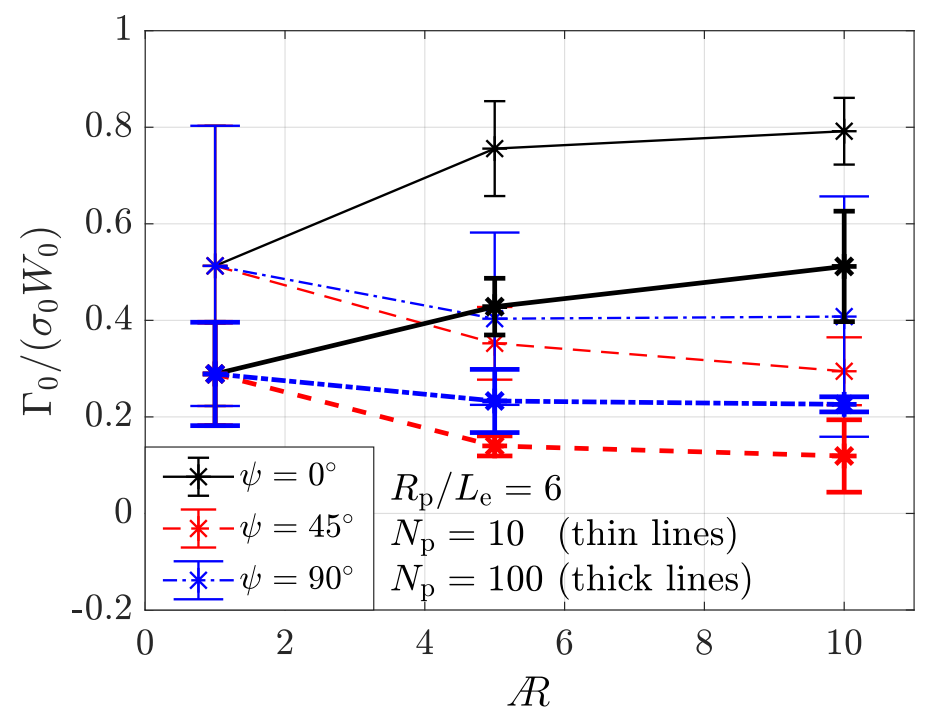

(a)

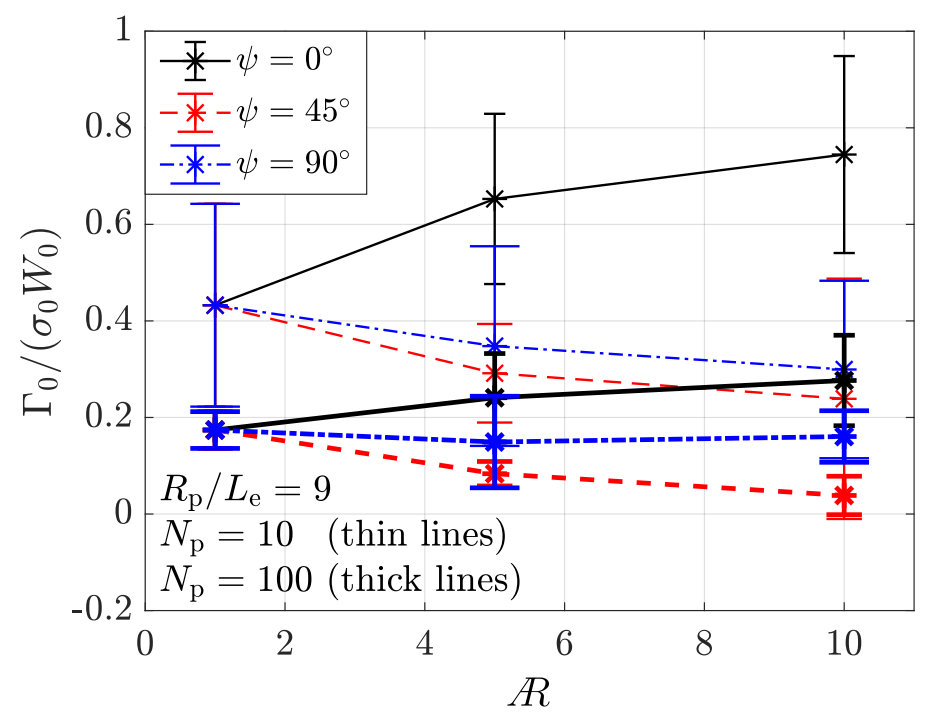

(b)

Figure 10: Tearing energy, $\Gamma_{0}$, as a function of the aspect ratio of the nucleation site, $R$, for a size of

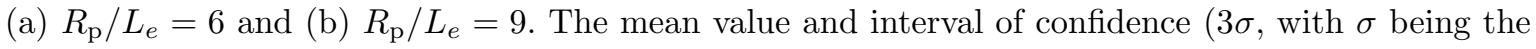
standard deviation), are indicated for $N_{\mathrm{p}}=10$ and $N_{\mathrm{p}}=100$. 


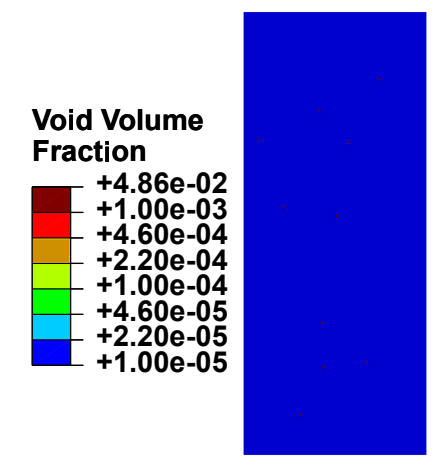

(a)

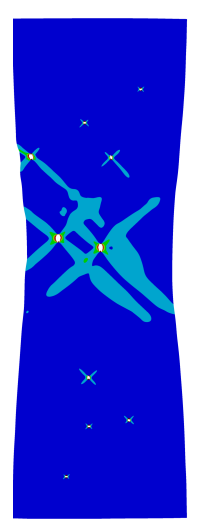

(b)

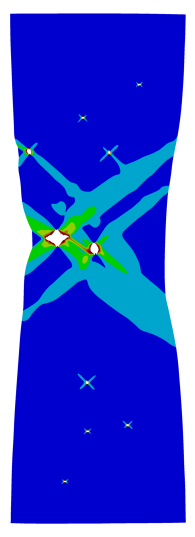

(c)

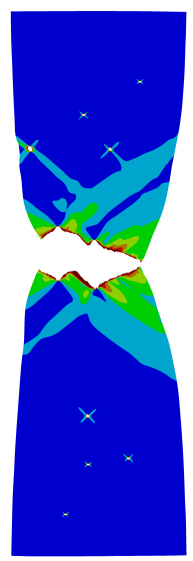

(d)

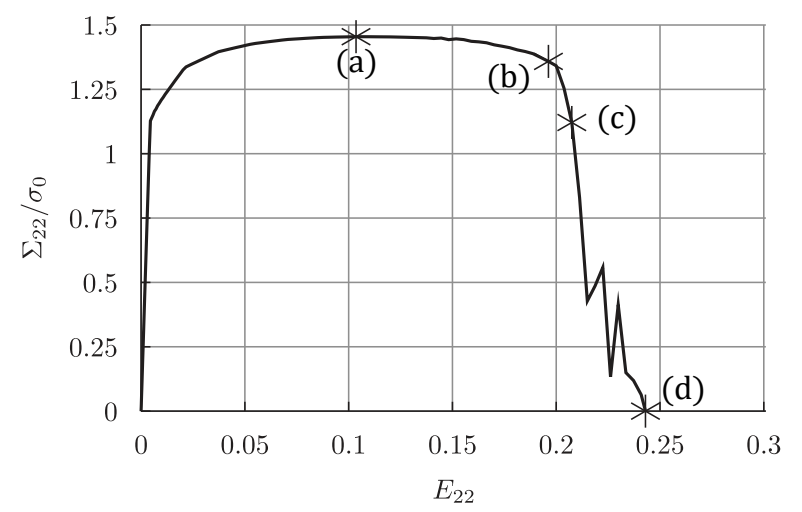

(e)

Figure 11: Developed void volume fraction in the fracture process zone for a plate with circular void nucleation sites and $N_{\mathrm{p}}=10-R_{\mathrm{p}} / L_{e}=3$, at the (a) onset of local thinning, (b) onset of macroscopic localization, (c) onset of microscopic localization, and (d) final failure. (e) Variation of the normalized engineering stress $\Sigma_{22} / \sigma_{0}$ with the engineering strain $E_{22}$ for the plate. 


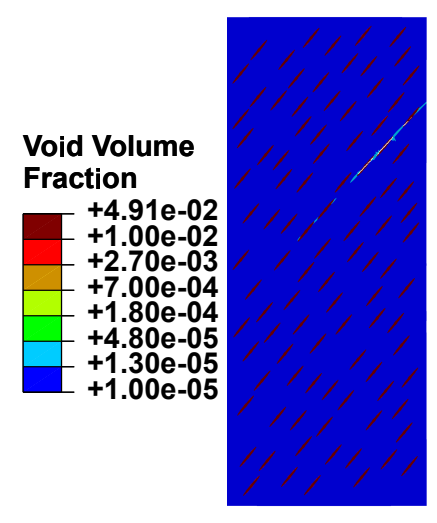

(a)

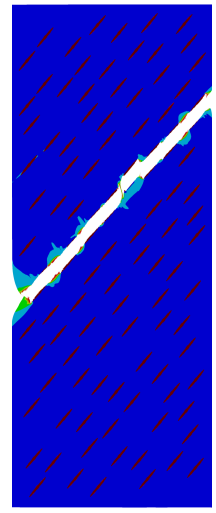

(b)

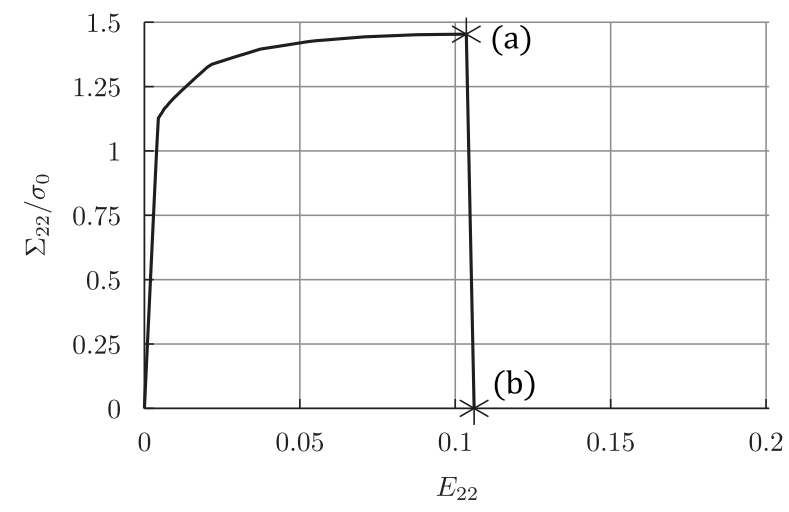

(c)

Figure 12: Developed void volume fraction in the fracture process zone for a plate with $N_{\mathrm{p}}=100$, $R_{\mathrm{p}} / L_{\mathrm{e}}=9, R=10$, and $\psi=45^{\circ}$, at the (a) onset of localized deformation, and (b) final failure. The three localized deformation stages (e.i. local thinning, macroscopic localization, and microscopic localization) set in simultaneously, unlike for the plate shown in 11. (c) Variation of the normalized engineering stress $\Sigma_{22} / \sigma_{0}$ with the engineering strain $E_{22}$ for the plate. 


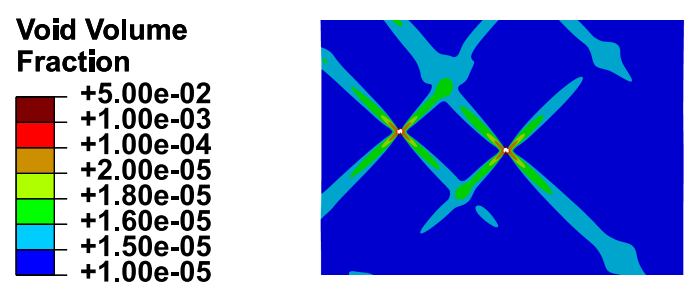

(a) Circular

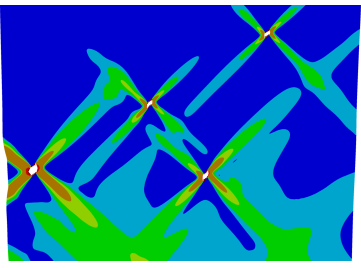

(c) $R=3, \psi=45^{\circ}$

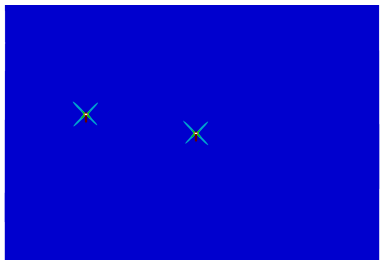

(b) $R=3, \psi=0^{\circ}$

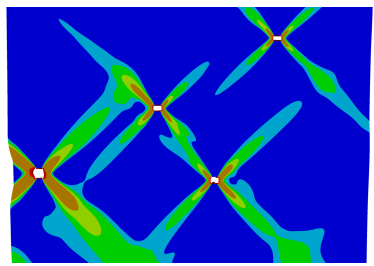

(d) $R=3, \psi=90^{\circ}$

Figure 13: Local shear bands emanating from the void nucleation sites and interaction mechanisms between the nucleation sites for four plates with $N_{\mathrm{p}}=10-R_{\mathrm{p}} / L_{e}=3$. The material and geometric parameters of the four plates, including the spatial distribution of the centers of the void nucleation sites are the same, except for the aspect ratio $(R)$ and orientation angle $(\psi)$ of the nucleation sites: (a) circular, (b) $R=3-\psi=0^{\circ}$, (c) $R=3-\psi=45^{\circ}$, and (d) $R=3-\psi=90^{\circ}$. The crack profiles for these four plates are displayed in Figs. $2(\mathrm{a}-\mathrm{d})$. 